\title{
Regional analysis of FDG and PIB-PET images in normal aging, mild cognitive impairment, and Alzheimer's disease
}

\author{
Yi Li • Juha O. Rinne $\cdot$ Lisa Mosconi • \\ Elizabeth Pirraglia $\cdot$ Henry Rusinek - Susan DeSanti • \\ Nina Kemppainen • Kjell Någren • Byeong-Chae Kim • \\ Wai Tsui • Mony J. de Leon
}

Received: 31 December 2007 / Accepted: 2 May 2008 / Published online: 20 June 2008

(C) Springer-Verlag 2008

\begin{abstract}
Objective The objective of the study is to compare the diagnostic value of regional sampling of the cerebral metabolic rate of glucose metabolism (MRglc) using [18F]-fluoro-2-deoxyglucose ([18F]FDG)-positron emission tomography (PET) and amyloid-beta pathology using Pittsburgh Compound-B ([11C]PIB)-PET in the evaluation of patients with Alzheimer's disease (AD) and mild cognitive impairment (MCI) compared to normal elderly (NL).

Materials and methods $\mathrm{AD}$ patients, $7 \mathrm{NL}, 13 \mathrm{MCI}$, and 17, received clinical, neuropsychological, magnetic resonance imaging (MRI), FDG, and PIB-PET exams. Parametric images of PIB uptake and MRglc were sampled using automated regions-of-interest (ROI).
\end{abstract}

Work at NYU was supported by NIH grants AG12101, AG08051, and AG13616. Work at Turku University was supported by the Academy of Finland, the Sigrid Juselius Foundation, and Turku University Hospital clinical grants (EVO).

Y. Li $\cdot$ L. Mosconi $\cdot$ E. Pirraglia $\cdot$ H. Rusinek $\cdot$ S. DeSanti $\cdot$

B.-C. Kim • W. Tsui $\cdot$ M. J. de Leon

New York University School of Medicine,

New York, NY, USA

Y. Li

e-mail: yi.li@med.nyu.edu

Y. Li

Qilu hospital Shandong University,

Jinan, China

J. O. Rinne $(\triangle) \cdot N$. Kemppainen $\cdot K$. Någren

Turku PET Centre, University of Turku,

FIN-20520, Turku, Finland

e-mail: juha.rinne@pet.tyks.fi
Results AD showed global MRglc reductions, and MCI showed reduced hippocampus (HIP) and inferior parietal lobe (IP) MRglc compared to NL. On PIB, AD patients showed significantly increased uptake in the middle frontal gyrus (MFG), posterior cingulate cortex (PCC), and IP ( $p \mathbf{s}<$ 0.05). PIB uptake in MCI subjects was either AD or NL-like. HIP MRglc and MFG PIB uptake were the best discriminators of NL from MCI and NL from AD. These two best measures showed high diagnostic agreement for AD (94\%) and poor agreement for MCI (54\%). For the NL vs. MCI discrimination, combining the two best measures increased the accuracy for PIB (75\%) and for FDG (85\%) to $90 \%$.

Conclusion For $\mathrm{AD}$, the pattern of regional involvement for FDG and PIB differ, but both techniques show high diagnostic accuracy and $94 \%$ case by case agreement. In the classification of NL and MCI, FDG is superior to PIB, but there is only $54 \%$ agreement at a case level. Combining the two modalities improves the diagnostic accuracy for MCI.

\section{B.-C. Kim}

Department of Neurology, Chonnam National University Medical School,

Gwangju, South Korea

W. Tsui • M. J. de Leon

Nathan Kline Institute,

Orangeburg, NY, USA

Y. Li • M. J. de Leon $(\bowtie)$

Center for Brain Health, Department of Psychiatry,

HN400 NYU School of Medicine,

560 First Avenue,

New York, NY 10016, USA

e-mail: mony.deleon@med.nyu.edu 
Keywords FDG-PET · PIB-PET · MRI · Alzheimer's disease $\cdot$ MCI $\cdot$ Diagnosis $\cdot$ Automated regions of interest

\section{Introduction}

Alzheimer's disease (AD) is an age-related neurodegenerative disorder that results in progressive loss of cognitive function. $\mathrm{AD}$ is characterized by the accumulation of the amyloid-beta $(\mathrm{A} \beta)$ peptide into amyloid plaques in the extracellular brain parenchyma and by intraneuronal neurofibrillary tangles caused by the abnormal phosphorylation of the tau protein [1]. Amyloid deposits and tangles are necessary for the post mortem diagnosis of $\mathrm{AD}$ [2].

Imaging techniques, such as positron emission tomography (PET), have long been used to visualize brain damage in $\mathrm{AD}$ and in mild cognitive impairment (MCI), often a prodrome to $\mathrm{AD}$ [3]. There is increasing evidence that reductions in the cerebral metabolic rate of glucose (MRglc), as measured with PET using [18F]-fluoro-2deoxyglucose (FDG) as the tracer, can be consistently detected in MCI patients compared to age-matched normal controls, mostly involving the parieto-temporal, posterior cingulate, and medial temporal cortices [4-7]. MRglc is an index of synaptic functioning and density [8], but hypometabolism is not specific to $\mathrm{AD}$, as it is observed in other neurodegenerative disorders (see [9] for review). Moreover, MCI is a clinical diagnosis in need of confirmatory biological evidence for disease. A recent large populationbased study showed up to $40 \%$ of patients with MCI who were subsequently diagnosed as cognitively normal [10].

The PET tracer, $N$-methyl[11C]2- (4'-methylaminophenyl)-6-hydroxy-benzothiazole, better known as Pittsburgh Compound-B (PIB), was used to detect amyloid deposition in vivo. Prior PIB-PET studies demonstrate quantitative increases in PIB uptake, reflecting greater amyloid burden, in $\mathrm{AD}$ and $\mathrm{MCI}$ patients compared to controls [11-14]. In AD, PIB uptake is particularly evident in the frontal, parieto-temporal, and posterior cingulate cortices, in keeping with the known distribution of amyloid plaques [15-17]. However, recent data also show that many MCI patients fall in between $\mathrm{AD}$ and control values for PIB binding, and some clinically normal subjects also show an elevated PIB uptake [13, 14]. These findings are also consistent with clinico-pathology studies showing that typical amyloid lesions are found in both demented and non-demented individuals $[18,19]$. These results suggest that the presence of amyloid may be necessary, though not sufficient for the symptoms consistent with the MCI stage of AD. The present study used a newly developed automated region of interest technique to compare the diagnostic value and concordance of FDG-PET and PIB$\mathrm{PET}$ in $\mathrm{AD}$ and $\mathrm{MCI}$.

\section{Materials and methods}

Subjects

Thirty-seven subjects, including: $17 \mathrm{AD}$ and $13 \mathrm{MCI}$ patients and 7 normal elderly (NL) patients, were examined at the University of Turku, Finland. All subjects underwent thorough clinical examinations including a medical history corroborated by a close informant, neurological and neuropsychological examinations, routine blood analysis, and magnetic resonance imaging (MRI). The AD patients were diagnosed according to the National Institute of Neurological and Communication Disorders and Stroke/ Alzheimer's Disease and Related Disorders Association (NINDS-ADRDA) [20] and DSM-IV criteria [21] by an experienced neurologist. Dementia severity was evaluated with the Mini-Mental State Examination (MMSE) [22]. All $\mathrm{AD}$ patients had progressive impairment of memory and impairment in at least one additional field of cognitive function.

All 13 MCI patients met the criteria for "amnestic MCI" [23]. The NL controls were healthy volunteers who contacted Turku PET Centre after announcement in newspaper or in public lectures concerning needed participation in memory studies. None of the controls reported or revealed on examination any neurological or psychiatric disease, prior head trauma, sensory impairment, or subjective cognitive complaints. The PET scans were used for the research evaluations and were not used for selection purposes. The study was approved by the Ethics Committee of Southwest Finland Health Care District.

\section{Brain imaging}

PET imaging All subjects underwent two PET scans, one with PIB and one with FDG on a GE Advance PET scanner (GE Medical Systems, Milwaukee, WI, USA) in the threedimensional scanning mode (septa retracted), yielding 35 slices with $4.25 \mathrm{~mm}$ thickness that covered the whole brain. The spatial resolution (full width at half-maximum) of the camera is $4.3 \mathrm{~mm}$ transaxially and $4.3 \mathrm{~mm}$ axially. Laser light beams were used for head positioning, with alignment determined by orbitomeatal and sagittal lines. Before the injection of either radiotracer, an 8-min transmission scan with ${ }^{68} \mathrm{Ge}$ rod sources was done for attenuation correction. All imaging data were reconstructed into a $128 \times 128$ matrix using a transaxial Hanning filter with a $4.6-\mathrm{mm}$ cutoff, and an axial ramp filter with an 8.5 -mm cutoff.

PIB-PET PIB-PET scans were acquired during a 90-min dynamic PET acquisition. [11C]PIB was injected into an antecubital vein as a bolus, with a mean dose of $382 \pm$ $103 \mathrm{MBq}$, and flushed with saline. The frame sequence of 
the PIB scan consisted of four 30-s frames, nine 1-min frames, three 3-min frames, ten 5-min frames, and two 10min frames. PIB-PET parametric images were obtained using the noninvasive Logan graphical analysis [24] using the 60- to 90 -min scans and the cerebellum as the reference region to estimate the PIB distribution volume ratios (DVR). This procedure has proved to be reliable and valid for PIB-PET studies in AD [25].

FDG-PET FDG-PET scans were acquired with a 55-min dynamic PET acquisition. During the uptake period, arterialized venous blood was sampled every $20 \mathrm{~s}$ for the first $3 \mathrm{~min}$, every $1 \mathrm{~min}$ from 3 to $5 \mathrm{~min}$, every $2.5 \mathrm{~min}$ from 5 to $10 \mathrm{~min}$, every $5 \mathrm{~min}$ from 10 to $35 \mathrm{~min}$, and every $10 \mathrm{~min}$ from 35 to $55 \mathrm{~min}$. The frame sequence of the FDG scan consisted of four 30-s frame, three 1-min frames, and ten 5-min frames. A Patlak plot was used to estimate the brain MRglc [26].

MRI MRI was performed with a Philips Gyroscan Intera $1.5 \mathrm{~T}$ CV Nova Dual scanner (Philips, the Netherlands). MRI included axial spin echo T2-weighted images $(\mathrm{TR}=$ $4488 \mathrm{~ms}$; TE $=100 \mathrm{~ms}$, slice thickness $=6 \mathrm{~mm}$, matrix $=512 \times$ $512)$, and $3 \mathrm{D}$ T1-weighted images $(\mathrm{TR}=25 \mathrm{~ms}, \mathrm{TE}=5 \mathrm{~ms}$, slice thickness $=1 \mathrm{~mm}$, matrix $=512 \times 512$ ).

\section{Image analysis}

All image processing and data analyses were performed at NYU blind to clinical diagnoses. MRI and PET scans were transferred to a Sun Sparc work-station (Sun Microsystems, Mountain View, CA, USA) where PIB- and FDG-PET scans were each co-registered with the corresponding MRI using a three-dimensional method based on minimizing the variance of the signal ratios [27], as implemented in the Multimodal Image Data Analysis System package (MIDAS, version 1.6) [28]. The implementation calls for a preliminary spatial alignment using intrinsic anatomical landmarks.

An MRI-based automated region of interest (ROI) technique was used to sample each individual's FDG and PIB images. The technique was validated by a manual ROI technique that is described in detail in the Appendix. The template ROI was first developed on seven MRI scans and then transferred to a co-registered MNI PET template. All PET scans were normalized to the PET template by a highorder polynomial transformation [29]. With spatial normalization parameters saved, an inverse transformation is applied to morph the ROIs back to the original FDG-PET. The standard FDG-ROI's are then transferred to the PIB scan in real space through the co-registration. ROI positioning was verified on the MRI, but no positioning adjustments were made in this project. To maximize gray matter $(\mathrm{GM})$ sampling, a probabilistic grey matter template image was derived from SPM [30] and added as template ROI (see Appendix). Nine MRI and FDG-PET validated automated ROIs were studied including: anterior putamen $(\mathrm{APu})$, grey matter (GM), hippocampus (HIP), inferior parietal lobe (IP), middle frontal gyrus (MFG), posterior cingulate cortex (PCC), cerebellum (C), superior temporal gyrus (STG), thalamus (TH).

The posterior lobe of the cerebellar cortex was used as the reference region for both PIB and FDG analyses. The cerebellum is minimally affected by either MRglc reductions $[4,31]$ or by amyloid pathology $[32,33]$ in AD.

\section{Statistical analysis}

The general linear model (GLM) univariate analysis of variance (ANOVA), with Tukey post hoc tests, was used to examine demographic, clinical, FDG MRglc, and PIB uptake measures across the three clinical groups. All significant results were confirmed using the nonparametric Mann-Whitney test with Bonferroni correction for multiple comparisons. Categorical demographic variables were examined with Chi-Square analysis and confirmed with Fisher's exact tests. PIB DVR is expressed as a ratio to cerebellar uptake. MRglc measures were adjusted for cerebellar MRglc as a covariate in the GLM. The bilateral regions showing the largest group effects (as determined by MANOVA) were examined with logistic regressions and ROC curves to assess their diagnostic accuracy in classifying the NL, MCI, and AD groups. The ROC curve was also used to determine optimal cutoff value for MRglc and PIB DVR in separating NL, MCI, and AD. Results were considered significant at $p<0.05$. All analyses were done using SPSS 12.0 (SPSS, Chicago, IL 2004, USA).

\section{Results}

\section{Clinical data}

The NL, MCI, and AD groups were comparable for age, gender, and education (see Table 1). The MMSE was significantly lower in $\mathrm{AD}$ subjects than in $\mathrm{NL}$ and $\mathrm{MCI}$ $(p<.05)$, but did not differ between MCI and NL. MMSE scores in the $\mathrm{AD}$ group ranged from 17 to 27, which corresponds to mild to moderate dementia.

\section{Group differences}

FDG-PET Of the nine regions tested, five regions showed significant post hoc differences between the NL and $\mathrm{AD}$ groups (Fig. 1, Table 2), with the $\mathrm{AD}$ group showing 
Table 1 Subjects' characteristics

\begin{tabular}{llll}
\hline Subjects & NL & MCI & AD \\
\hline$N$ & 7 & 13 & 17 \\
Age in years [range] & $69.1(5.4)$ & $71.6(4.6)$ & $72(4.7)$ \\
& {$[59-74]$} & {$[64-80]$} & {$[59-79]$} \\
Education (years) & $9.9(2.9)$ & $10.8(3.8)$ & $9.7(4.0)$ \\
Mini Mental State & $28.9(0.9)$ & $27.6(1.4)$ & $23.6(2.9) * * *$ \\
Exam [range] & {$[28-30]$} & {$[25-29]$} & {$[17-27]$} \\
Gender (\% female) & $71 \%$ & $31 \%$ & $53 \%$ \\
\hline
\end{tabular}

Values are mean (SD).

*Significantly different from NL, $p<0.05$

**Significantly different from MCI, $p<0.05$

reduced MRglc compared to NL in the HIP (43\%), PCC (21\%), IP (18\%), MFG (13\%), ( $p$ s $<.05)$. Significant MRglc reductions in MCI compared to NL were found for the HIP $(16 \%)$ and IP (13\%), and in AD compared to MCI reductions, which were only found in the HIP (23\%; $p$ 's $<.05)$. For all group comparisons, the HIP MRglc was the most significant group discriminator $\left(F_{[2,34]}=33.9\right.$, $p<.001)$. No cerebellar differences were observed for any group comparisons.

PIB-PET AD patients showed significantly higher PIB uptake compared to both $\mathrm{NL}$ and $\mathrm{MCI}$ groups $\left(F_{[2,34]}=\right.$ 14.6, $p<.005)$, respectively, in the GM, MFG, PCC, IP, STG (Fig. 2, Table 3). The MFG was the region showing the highest PIB uptake $\left(F_{[2,34]}=14.6, p<.001\right)$, which was $66 \%$ higher in AD compared to NL $(p=.0001)$, and $29 \%$ higher compared to MCI $(p=.004)$. There is no significant differences found between NL and MCI; however, the $\mathrm{APu}$ region showed a trend for higher PIB uptake in MCI $(p=.06)$. No cerebellar differences were observed for any group comparisons.

PIB-FDG associations Negative correlations between the FDG and PIB modalities were observed when the three groups were combined: IP $(r=-0.43, p=.001)$, STG $(r=-0.41$,
Table 2 FDG-PET MRglc data by diagnostic group

\begin{tabular}{llll}
\hline Region & NL & MCI & AD \\
\hline APu & $28.4(1.31)$ & $26.7(0.98)$ & $26.1(0.86)$ \\
GM & $21.6(0.63)$ & $19.7(0.47)$ & $19.2(0.41)^{*}$ \\
HIP & $20.4(0.79)$ & $17.6(0.59)^{*}$ & $14.3(0.52)^{*}, * *$ \\
IP & $23.7(0.87)$ & $21.0(0.65)^{*}$ & $20.1(0.57)^{*}$ \\
MFG & $26.0(0.99)$ & $23.2(0.75)$ & $23.0(0.65)^{*}$ \\
PCC & $25.8(0.98)$ & $23.0(0.74)$ & $21.4(0.64)^{*}$ \\
STG & $22.2(0.96)$ & $20.0(0.72)$ & $19.8(0.63)$ \\
TH & $27.9(1.42)$ & $26.9(1.06)$ & $25.7(0.93)$ \\
\hline
\end{tabular}

Values are mean (SD) of covariate adjusted values.

$A P u$ Anterior putamen, $G M$ gray matter, $H I P$ hippocampus, $I P$ inferior parietal lobule, $M F G$ middle frontal gyrus, $P C C$ posterior cingulate cortex, $S T G$ superior temporal gyrus, $T H$ thalamus

* Significantly different from NL, $p<0.05$

**Significantly different from MCI, $p<0.05$

$p=.001)$, and PCC $(r=-0.40, p=.001)$. However, no significant intraregional correlations were observed within any of the three diagnostic groups.

As shown in Fig. 3, the FDG-PET and PIB-PET data show different regional patterns of involvement in $\mathrm{AD}$ compared with NL. The most significantly affected region on FDG was the hippocampus and the middle frontal gyrus on PIB (Fig. 4).

Diagnostic models

Logistic regression models were used to examine regional uptake and FDG-MRglc as predictors of group membership. Diagnostic classification accuracy, sensitivity (SS), and specificity (SP) are found in Table 4.

$A D$ vs. NL HIP MRglc yielded an accuracy of $92 \%\left(X^{2}{ }_{[1]}=\right.$ 21.8, $p<.001, \mathrm{SS}=100, \mathrm{SP}=88)$. MFG PIB uptake distinguished AD from NL with $96 \%$ accuracy $\left(X_{[1]}^{2}=29.0\right.$, $p<.001, \mathrm{SS}=94, \mathrm{SP}=100$ ). Using a PIB-DVR cutoff of 1.4 , three regions including the MFG, PCC, and IP each separated 16 out of $17 \mathrm{AD}$ and 7 out of 7 NL with $94 \%$
Fig. 1 Scatter plots showing regional cerebellar adjusted MRglc (umol $/ 100 \mathrm{~g} / \mathrm{min}$ ) for the $H I P, M F G, I P$, and PPC in $N L$, $M C I$, and $A D$ groups. The horizontal lines show the group means

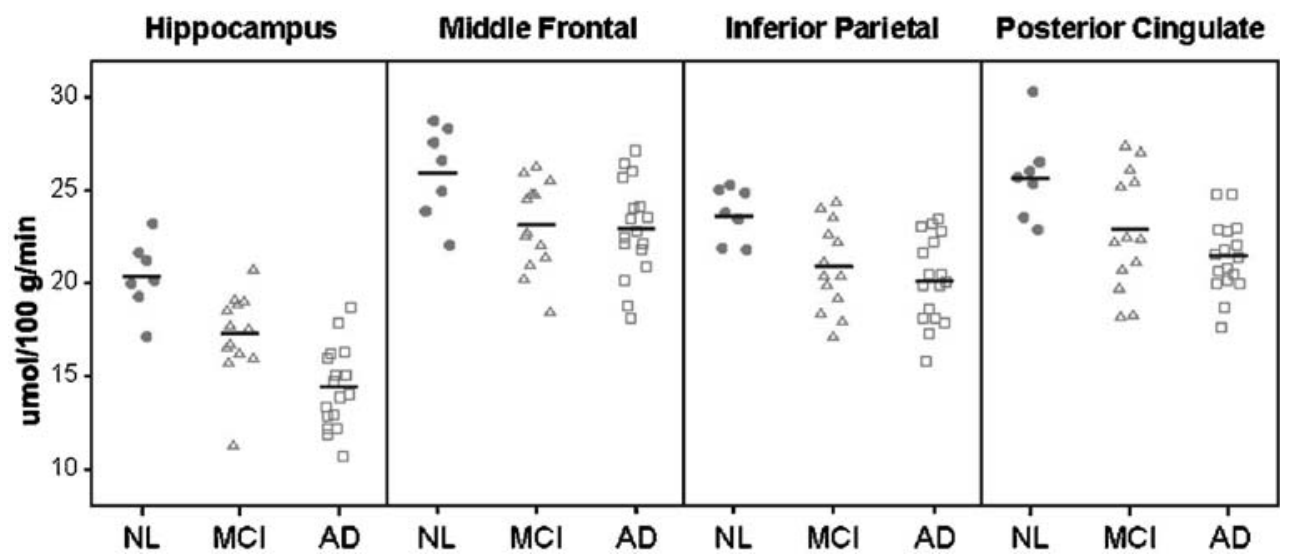


Fig. 2 Scatter plots showing regional PIB binding from the ROI analysis in the hippocampus, MFG, IP, and PPC in subjects with $N L, M C I$, and $A D$. The small horizontal lines show the groups mean values. The large horizontal line shows for reference purposes the DVR set to 1.4

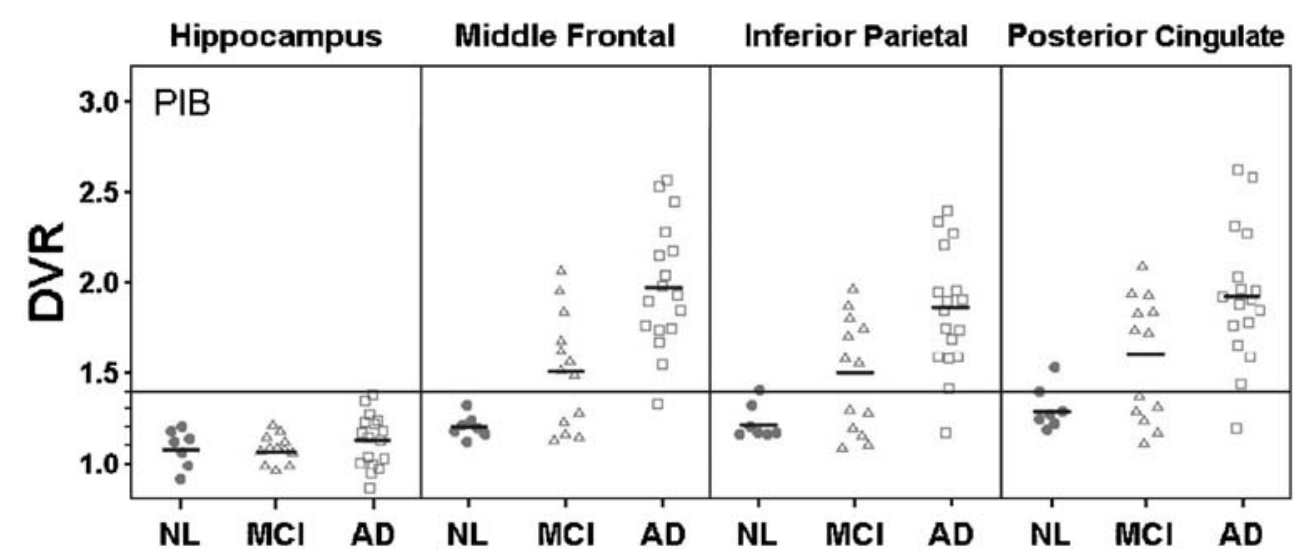

sensitivity and $100 \%$ specificity with an overall accuracy of $96 \%$. The one erroneously classified as AD subject had the PIB-DVR lower than 1.4 in all three regions (Fig. 2). Comparing PIB-MFG and FDG-HIP, there was high diagnostic agreement for the classification of $\mathrm{AD}(94 \%)$ and NL (86\%) subjects.

MCI vs. NL The accuracy for the HIP MRglc in distinguishing MCI from NL was $85 \%\left(X_{[1]}^{2}=9.2, p<.01\right.$, $\mathrm{SS}=$ $85, \mathrm{SP}=86$ ), and for MFG PIB uptake, the accuracy was $75 \%\left(X_{[1]}^{2}=7.2, p<.01, \mathrm{SS}=62, \mathrm{SP}=100\right)$. Thus, about $60 \%$ of the MCI subjects showed an AD-like DVR $>1.4$; see Fig. 5). Comparing PIB-MFG and FDG-HIP for the classification of MCI showed, there was poor agreement (54\%). Only 7 out of 13 MCI cases showed both high PIB binding and low MRglc. Combining the MFG PIB and HIP-FDG measures improved the classification of NL and MCI to $90 \%$ (increment $X^{2}{ }_{[1]}=4, p<.05, \mathrm{SS}=92, \mathrm{SP}=86$ ). In an effort to relate these MCI data to the clinical findings, we examined the association between MFG PIB uptake and MMSE scores. All lower MMSE 25-27 scoring MCI patients were AD-like (PIB DVR>1.4) and all NL-like MCI (PIB DVR $<1.4$ ) had MMSE scores $>28$ (see Fig. 3, $\left.t=2.7_{[1,11]}, p<0.05\right)$. There were no regional MRglc differ- ences between the high and low MMSE MCI group. As mentioned above, the MCI patients typically showed low HIP MRglc. Graphic depiction of the MFG-PIB and HIPFDG relationships by MMSE score is found in Fig. 5. These data show that all lower MMSE 25-27 scores, MCI patients are in the high PIB binding and low MRglc quartile. For the low MMSE group, there was $100 \%$ agreement between PIB and FDG, as opposed to 33\% for high MMSE group.

$A D$ vs. $M C I$ MFG PIB uptake yielded an overall accuracy of $77 \%$ in distinguishing $\mathrm{AD}$ from $\mathrm{MCI}\left(X_{[1]}^{2}=8.9, p<.01\right)$. HIP MRglc yielded an accuracy of $80 \%\left(X^{2}{ }_{[1]}=11.6\right.$, $p<.01)$. The combination of MFG PIB uptake and HIP MRglc improved the classification of $\mathrm{AD}$ and MCI to $83 \%$ (increment $X_{[1]}^{2}=4.1, p<.01, \mathrm{SS}=82, \mathrm{SP}=85$ ).

\section{Discussion}

The uptake of the beta amyloid PET tracer [11C]PIB was significantly increased in $\mathrm{AD}$ compared to age-matched healthy controls. This effect was found bilaterally in the middle frontal gyrus, anterior putamen, inferior parietal

Table 3 PIB-PET DVR data by diagnostic group

\begin{tabular}{|c|c|c|c|c|c|}
\hline Region & NL & MCI & MCI:NL ratio & $\mathrm{AD}$ & $\mathrm{AD}: \mathrm{NL}$ ratio \\
\hline $\mathrm{APu}$ & $1.21(0.11)$ & $1.67(0.50)$ & 1.38 & $1.99(0.42)^{*}$ & 1.64 \\
\hline GM & $1.12(0.09)$ & $1.29(0.22)$ & 1.15 & $1.54(0.24)^{*}, * *$ & 1.38 \\
\hline HIP & $1.03(0.11)$ & $1.02(0.11)$ & 0.99 & $1.08(0.16)$ & 1.05 \\
\hline IP & $1.19(0.12)$ & $1.48(0.35)$ & 1.24 & $1.82(0.35)^{*}, * *$ & 1.53 \\
\hline MFG & $1.16(0.09)$ & $1.50(0.36)$ & 1.29 & $1.92(0.36)^{*}, * *$ & 1.66 \\
\hline PCC & $1.26(0.14)$ & $1.58(0.38)$ & 1.25 & $1.93(0.39)^{*}, * *$ & 1.53 \\
\hline STG & $1.15(0.13)$ & $1.36(0.32)$ & 1.18 & $1.67(0.30)^{*}, * *$ & 1.45 \\
\hline $\mathrm{TH}$ & $1.38(0.12)$ & $1.44(0.30)$ & 1.04 & $1.64(0.23)$ & 1.19 \\
\hline
\end{tabular}

*AD Significantly different from NL, $p<0.05$

**AD Significantly different from MCI, $p<0.05$ 

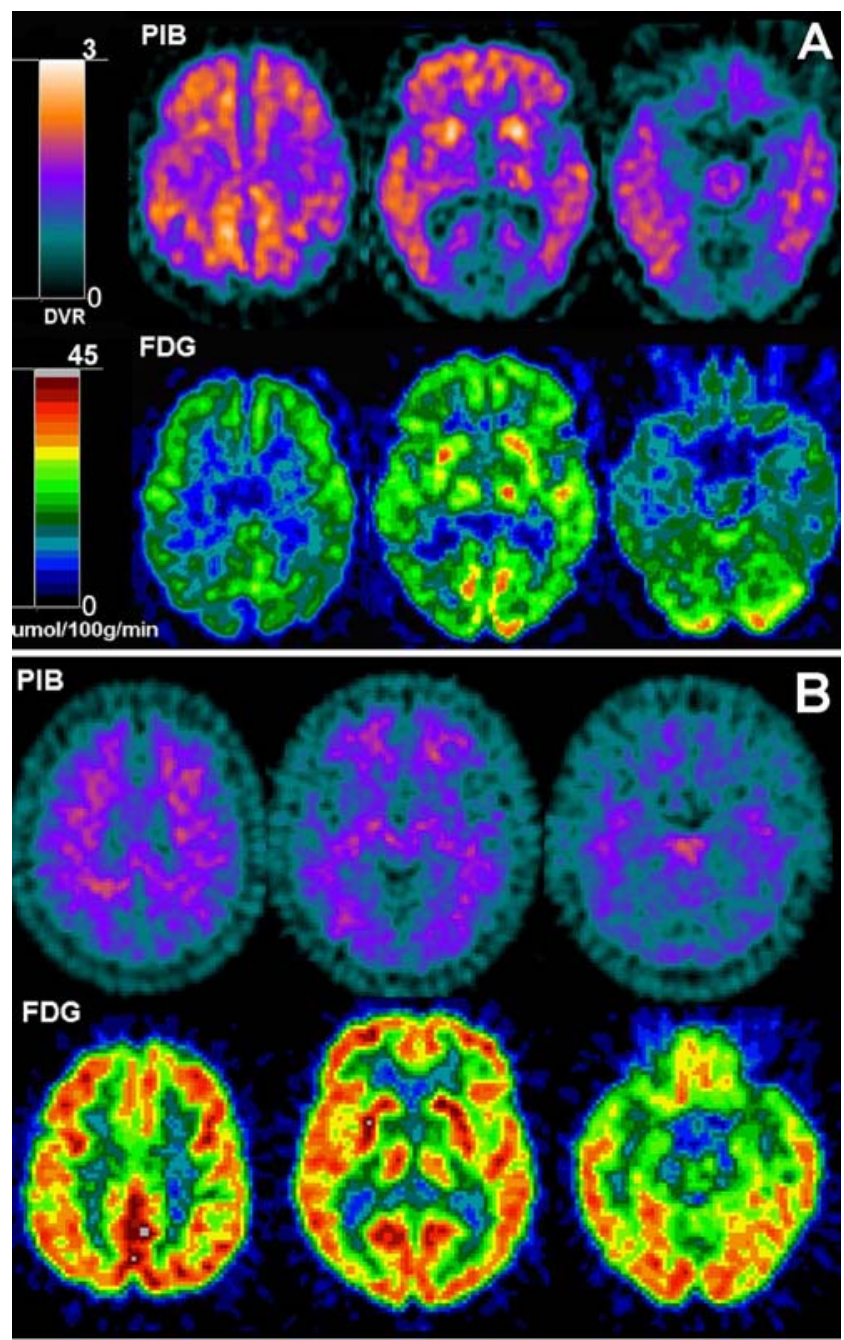

Fig. 3 PIB and FDG-PET scans from two representative subjects: a A 71-year-old male AD subject, GDS 5, MMSE 19; b a 65-year-old male NL subject, GDS 1, MMSE 29. Top row: PIB-PET images; bottom row: co-registered FDG-PET images. PET scans are displayed in the axial plane, from the top to the bottom of the brain, at the level of the centrum-semiovale (left), basal ganglia (center), and medial temporal lobe (right)

lobule, and the posterior cingulate cortex. In MCI, a lesser pattern of PIB uptake was found involving the middle frontal gyrus and inferior parietal lobule. This observation is also consistent with findings reported in previous studies $[14,34]$. The FDG-PET data demonstrated that AD patients show a pattern of bilateral MRglc reductions in the hippocampus, posterior cingulate, inferior parietal, and frontal cortices, while MCI patients presented with hypometabolism most consistently in the hippocampus and in the parietal cortex. These findings are also consistent with prior FDG-PET studies [6, 7, 37, 38, 39, 40].

The MFG PIB uptake separated 16 out of 17 AD patients from NL control with $100 \%$ specificity and $96 \%$ sensitivity. This contributes to the view that [11C]PIB-PET will have utility as a diagnostic marker for AD. Only one 73-year-old male AD patient [Global Deterioration Scale (GDS) 5, MMSE 19] showed low PIB retention (DVR=1.29). The finding of occasional "PIB negative" AD patients has been previously reported $[14,35]$, and the reason is unclear. Such findings require post mortem clarification. On the other hand, the FDG-PET scan of this $\mathrm{AD}$ patient showed evidence for a neurodegenerative disorder consistent with $\mathrm{AD}$, as reflected in the bilateral MRglc reductions in the parieto-temporal posterior cingulate cortices and medial temporal lobes. While direct diagnostic comparison between PIB and FDG imaging is uncommon, a previous study [36] reported PIB-PET to be superior to FDG-PET in classifying AD from NL. Unlike our study, this paper only studied neocortical regions and not hippocampus, which we found most discriminative on FDG-PET. Our study shows that the best regions for FDG-PET (hippocampus) and PIBPET (middle frontal gyrus) have high diagnostic agreement for $\mathrm{AD}(94 \%)$ and $\mathrm{NL}(86 \%)$ indicating approximately equal value in the clinical diagnosis of AD. The combination of two PET modalities did not improve the diagnostic discrimination between $\mathrm{AD}$ and NL. That there was no appreciable increase in the classifications of $\mathrm{AD}$ and $\mathrm{NL}$, is attributable to the very high accuracy each modality achieved on its own.

We found that several PIB regions were found to discriminate between MCI and NL with accuracies in the 60-75\% range compared with FDG regions that performed in the $70-85 \%$ range. FDG-PET appears to be superior to PIB-PET in the classification of NL and MCI. Moreover, the diagnostic agreement between the two PET modalities for MCI was only 54\%; four MCI subjects with MRglc reductions showed low PIB retention and one MCI subject with normal MRglc showed high PIB retention. With MCI subjects separated into high PIB uptake (AD-like) and low PIB uptake (NL-like) groups (Fig. 5), we found that the MCI subjects with high MMSE scores $(\geqq 28)$ have NL-like PIB scans and MCI subjects with low MMSE scores have AD-like PIB scans. Moreover, elevated PIB and low MRglc was found in $100 \%$ of low performing MCI compared with only $33 \%$ agreement for the high performing MCI. These data suggest that low performing MCI subjects with PIB positive scans are at increased risk for dementia. Overall, the data show that the combination of two PET modalities improves the diagnostic discrimination between MCI and NL. Longitudinal studies are needed to clarify the utility of the PIB and FDG-PET imaging in assessing risk for AD.

Our results comparing $\mathrm{MCI}$ and $\mathrm{AD}$ showed different patterns of regional involvement depending on the PET imaging modality. We observed that the mean PIB values for several regions differed between $\mathrm{MCI}$ and $\mathrm{AD}$ and that their diagnostic classifications were significant. Consistent diagnostic PIB effects ( $70 \%$ accuracy) were found in the middle frontal gyrus, posterior cingulate, inferior parietal 
Fig. 4 Mean $M C I$ and $A D$ $Z$-scores relative to $N L$. The most significantly affected region for FDG is the hippocampus and for PIB, frontal neo-cortex

\section{Mean $\mathrm{MCl}$ and $\mathrm{AD} \mathrm{Z}$-scores Relative to $\mathrm{NL}$}

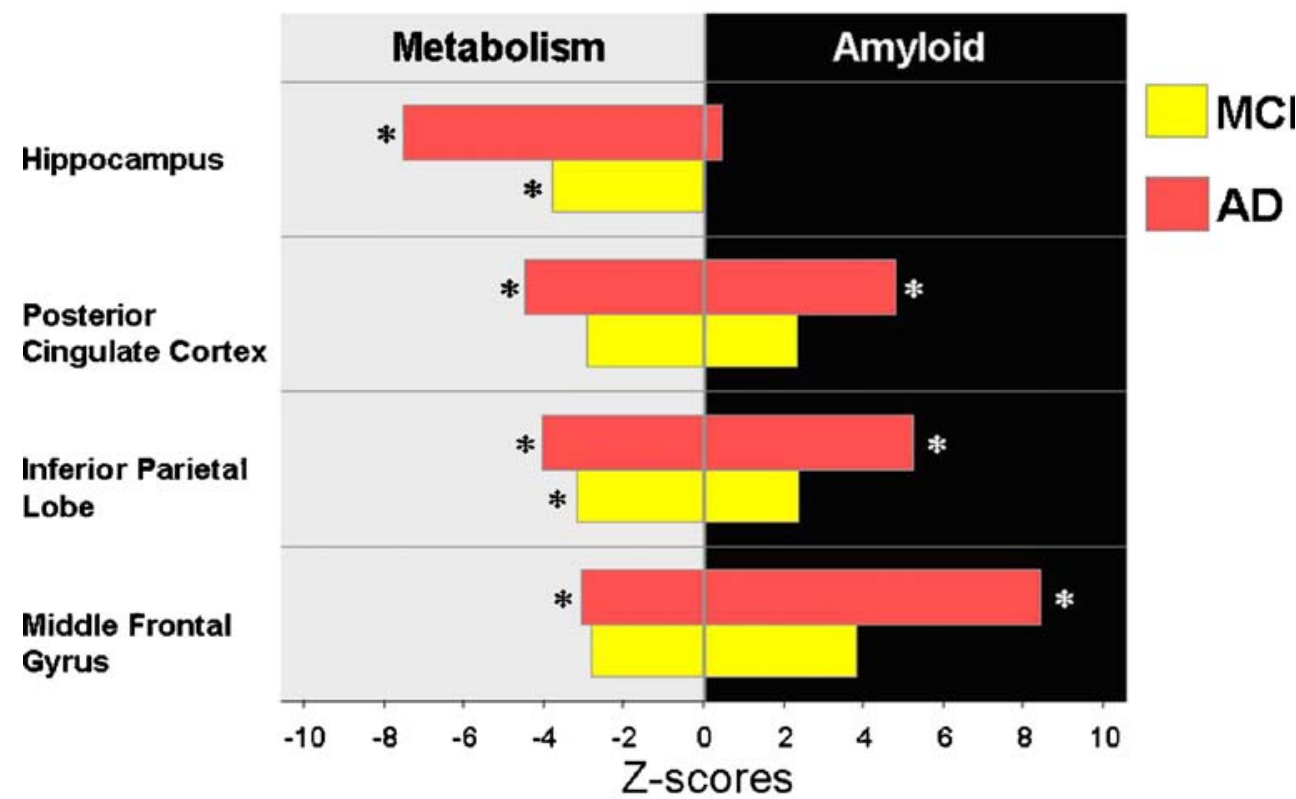

Sig. difference from NL $(p \leq .05)$ lobule, and the superior temporal gyrus. For FDG, the hippocampus, the only region that showed a mean MRglc reduction in $\mathrm{AD}$ relative to $\mathrm{MCI}$ also showed the only significant diagnostic effects $(\sim 80 \%)$. This result of modality-specific informative regions underlies our second example where the combination of the two PET techniques yields complementary information in the detection of pathology. In our study, the combination of the two PET modalities improved the diagnostic discrimination between $\mathrm{MCI}$ and $\mathrm{AD}$ and between MCI and NL.

We did not observe an inverse relationship between the regional PIB and FDG data in $\mathrm{AD}$ as reported by others $[14,35]$. This discrepancy may be in part due to the statistical designs used to study the data. Klunk et al

Table 4 Diagnosis classification accuracy, sensitivity, and specificity (\%) of PIB-PET and FDG-PET

\begin{tabular}{|c|c|c|c|c|c|c|c|c|c|}
\hline \multirow[t]{2}{*}{ Region } & \multicolumn{3}{|c|}{ NL vs. AD } & \multicolumn{3}{|c|}{ NL vs. MCI } & \multicolumn{3}{|c|}{ MCI vs. AD } \\
\hline & A & SS & SP & A & SS & SP & A & SS & SP \\
\hline \multicolumn{10}{|c|}{ PIB-PET } \\
\hline $\mathrm{APu}$ & & & & 70 & 62 & 86 & - & - & - \\
\hline HIP & 96 & 94 & 100 & - & - & - & - & - & - \\
\hline IP & - & - & - & 70 & 54 & 100 & 73 & 94 & 54 \\
\hline MFG & 96 & 94 & 100 & 75 & 62 & 100 & 70 & 94 & 62 \\
\hline PCC & 96 & 94 & 100 & 60 & 54 & 71 & 73 & 94 & 54 \\
\hline STG & 88 & 94 & 71 & - & - & - & 67 & 65 & 69 \\
\hline \multicolumn{10}{|c|}{ FDG-PET } \\
\hline $\mathrm{APu}$ & - & - & - & - & - & - & - & - & - \\
\hline HIP & 92 & 88 & 100 & 85 & 85 & 86 & 80 & 76 & 85 \\
\hline IP & 83 & 88 & 71 & 75 & 77 & 71 & - & - & - \\
\hline MFG & 79 & 76 & 86 & 80 & 92 & 57 & - & - & - \\
\hline PCC & 88 & 88 & 86 & 70 & 62 & 86 & - & - & - \\
\hline STG & 79 & 82 & 71 & 70 & 69 & 71 & - & - & - \\
\hline
\end{tabular}

The most significant region was labeled by bold font and "-" stand for nonsignificant.

A Accuracy, $S S$ sensitivity, $S P$ specificity, $A P u$ anterior putamen, $H I P$ hippocampus, $M F G$ middle frontal gyrus, $I P$ inferior parietal lobe, $P C C$ posterior cingulate cortex, $S T G$ superior temporal gyrus 


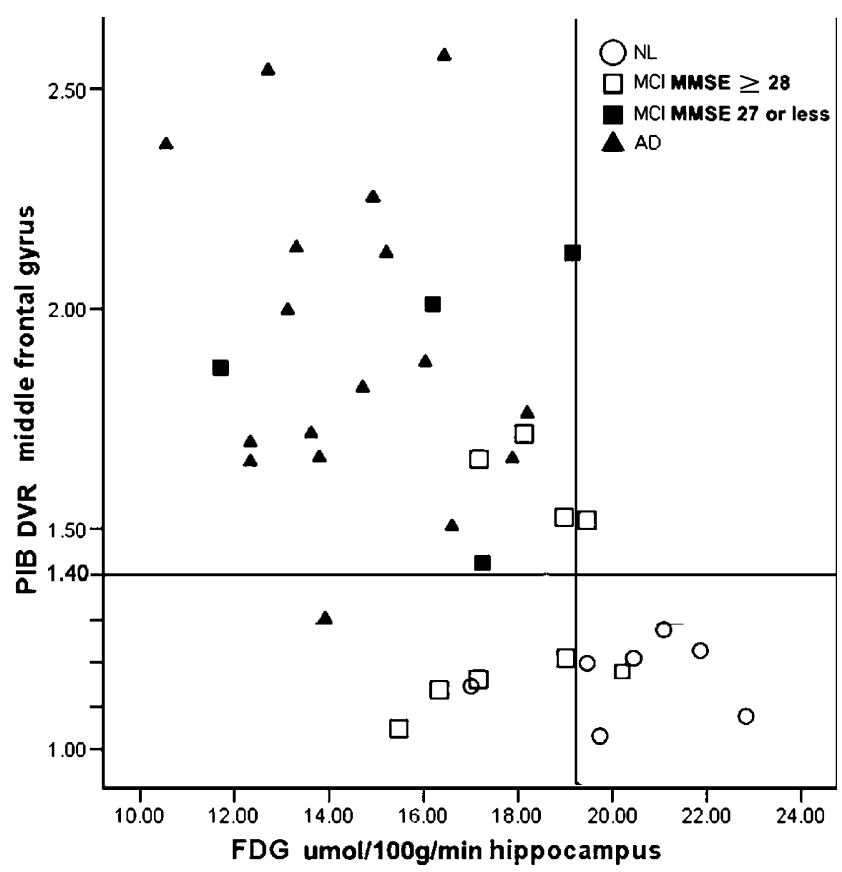

Fig. 5 Scatter plots showing the combined use of $P I B$ of MFG and FDG of HIP on the classification of the $N L, M C I$, and $A D$ groups

demonstrated a significant correlation in the inferior parietal cortex in a combined group of $\mathrm{AD}$ and NL patients. However, they observed that this effect did not remain significant when only AD patients were studied. [14]. While Edison et al. showed lower CMRglc values correlated with higher PIB uptake ratios in temporal $(p=.05, r=-0.58)$ and parietal lobes $(p=.041, r=-0.60)$ in 12 AD subjects, they also observed a high frontal amyloid load in the face of spared glucose metabolism. These preliminary results suggest that the weak inverse correlations observed may be due to either group diagnostic effects or to different regional metabolic vulnerability due to the complex neuropathology of AD. Overall, it appears that amyloid plaques may not be directly responsible for neuronal dysfunction in $\mathrm{AD}$.

In the present study, we describe the continued development of an automated ROI technique custom-tailored for FDG and PIB-PET images (see Appendix for technical details). Several automated tools are used in neuroimaging studies to examine and sample brain regions. Foremost, voxel-based analysis (VBA) techniques with statistical parametric mapping procedures provide examination of statistical effects through the whole-brain on a voxel-byvoxel basis [41, 42]. The basic procedure in VBA involves the spatial normalization and smoothing of each individual PET scan to a spatially standardized brain reference image (i.e., the "template" image) in the stereotactic space, thus enabling automated voxel-by-voxel assessment of statistical effects [41, 42]. However, the MRI-guided ROI technique remains the gold standard for PET sampling, especially in aging and degenerative diseases, because of its superior anatomical precision. On the other hand, the conventional manual ROI sampling is time consuming and operator dependent, and PET images are often acquired without a corresponding 3D research MRI. To examine large data sets with reasonable anatomical precision, we describe the development of an automated ROI technique customtailored for sampling the cortical and medial temporal lobe regions affected in $\mathrm{AD}$ on FDG and PIB-PET images. These procedures were validated against the gold-standard manual ROI determined on the co-registered MRI scans. The automated ROI data in this project showed high anatomical precision as assessed on the MRI scans and high agreement with respect to the manual MRglc sampling $(r$ 's $>0.90)$ and manual PIB sampling ( $r$ 's $>0.90$; detail was described in Appendix). In the present study, the anatomical accuracy of the automated ROIs was excellent in all subjects, and positional adjustments were not made.

Our automated method offers several advantages compared to other commonly used image analysis tools. First, the ROIs are applied via inverse transformation to the original image instead of the spatially normalized image, thus preserving the anatomical shape of the region and avoiding possible sampling errors. Moreover, the anatomical precision of the ROIs can be directly examined on the MRI and PET scans in the original space, and manual adjustments can be made if necessary. Furthermore, we applied a sampling strategy to optimize gray matter sampling, which minimizes partial volume effects and nonspecific white matter binding, which may confound detection of regional changes on PIB-PET.

There are some limitations in this study. First, the recruitment of patients at a university-based unit limits the generalization of the results. Second, we used a probabilistic gray matter sampling technique instead of the traditional MRI-based atrophy correction. While our tests suggest comparability between the two techniques, there remains the possibility that it may not remove partial volume effect thoroughly. Third, this study relied on cross-sectional data where longitudinal follow-up studies are needed to determine the predictive accuracy of PIB-PET and FDG-PET in the $\mathrm{MCI}$ progression to $\mathrm{AD}$.

\section{Conclusion}

We developed and applied an automated image analysis system for the regional sampling of PIB and FDG PET scans in a cross-sectional study of normal elderly, MCI, and AD. We observed widespread cortical amyloid deposition and widespread reductions in brain glucose metabolism in $\mathrm{AD}$ patients that were of equivalent high value in diagnosis. For MCI, the regional patterns were less prominent. For 
both the $\mathrm{AD}$ and the MCI groups, the diagnostically most useful PIB region was the middle frontal gyrus, and the most useful MRglc reductions were found in the hippocampus. For MCI, the two modalities were diagnostically inconsistent, and this contributed to their combined added value. Little evidence was found for an inverse relationship between regional PIB uptake and reduced MRglc. Longitudinal studies are needed to clarify the early and relative predictive utility of PIB and FDG-PET imaging in assessing the risk for progressive cognitive decline among MCI patients and for assessing probable $\mathrm{AD}$ patients without significant PIB uptake.

Conflicts of interest J.R. is a former part-time consultant of Turku Imanet, a subsidiary of GE Healthcare. LM, HR, WT, and MdeL have patents and licensing agreements pending on the HipMask image analysis software used in this paper.

\section{Appendix}

\section{Summary}

We previously developed and validated an automated ROI procedure for the hippocampus [1]. Below, we describe the development of an automated cortical regions-of-interest (ROI) technique custom-tailored for FDG and PIB-PET images in NL and AD subjects. The template ROI was first developed on seven MRI scans and then transferred to a coregistered MNI PET template. The technique was validated against the gold-standard manual ROI approach and shows high anatomical precision as assessed on MRI scans and high sampling accuracy with respect to the manual MRglc sampling. This technique enables one to regionally sample individual brain PET scans in an automated fashion with high anatomical precision without an MRI scan. In brief, an individual subject's FDG-PET image is first spatially normalized to the standard FDG-PET template by a highorder polynomial transformation [2]. The reverse of this transformation is then applied to the ROIs on the template to map them back to sample the original FDG-PET image [3]. While the technique we developed does not require an MRI, with the availability of a co-registered MRI, the positional accuracy of the sample site can be individually verified. This program was implemented in the NYU MIDAS image analysis software package [3].

Development of anatomy-validated standardized regions of interest

ROIs were manually drawn on the Montreal Neurological Institute (MNI) single-subject MRI template [4], based on specific anatomical landmarks (such as brain fissures and sulci), using published protocols with known intra-rater reliability higher than $90 \%[5,6,7]$. The ROIs were then transferred to six spatially normalized MRI scans [three elderly normal (NL) and three Alzheimer disease (AD) patients]. The mean positions of the boundary sulci from the seven subjects were determined and used to determine the final "probabilistic" ROI boundaries [8]. This has the effect of reducing the anatomical variation of the template ROIs [9].

The MNI single-subject MRI template image was resampled on a $105 \times 126 \times 91$ matrix with an isotropic voxel size of $1.5 \times 1.5 \times 1.5 \mathrm{~mm}$. The template ROIs defined included: prefrontal cortex $(\mathrm{PF})$, middle frontal gyrus (MFG), superior temporal gyrus (STG), inferior parietal lobule (IP), posterior cingulate cortex (PCC), occipital lobe (OL), cerebellum (C) (see Fig. 1). The hippocampus (HIP), thalamus $(\mathrm{T})$, and anterior putamen $(\mathrm{APu})$ were sampled using the previously published Hipmask technique [1].

To maximize grey matter (GM) sampling, each ROI was further accommodated in size according to a priori grey matter template image [10]. This a priori GM image is a probabilistic image of MRI-based GM distribution in the general population [10], with intensity in each voxel representing the probability that the voxel includes GM. On this probabilistic GM image, only voxels with GM probability $>90 \%$ were retained, and each ROI was restricted in size to match the new defined probabilistic boundaries. Furthermore, to limit partial volume CSF effects at the gray matter ROI boundaries, each ROI was eroded by 2 pixels $(3.0 \mathrm{~mm})$ in all directions.

Consequently, the template ROIs were used to sample MRglc and PIB for all subjects by applying an inverse transformation that morphs all ROIs back to the original PET images while preserving the anatomical shape of the region.

\section{Sampling the PET Scans}

PET scans were normalized to the MNI PET template space to obtain the transformation parameters. This protocol avoids the necessity of having to have MRI scans to undertake an automated analysis. Because the alternative normalization of an individual MRI to an MRI template, with the subsequent transferring normalization parameters to a corresponding PET image, may provide a higher spatial accuracy than normalization of a PET image to a PET template image, the two normalization methods were tested. The ROI template program was used to compare the MRI vs. PET normalizations. We observed high correlations between the two normalization methods: for FDG-PET, the $r$ 's ranged from 0.85 (HIP) to 0.98 (IP) $(p$ 's $<.001)$, and for PIB-PET the $r$ 's ranged from 0.87 (STG) to 0.92 (IP) $(p$ 's $<.001)$. 
Fig. 6 a Template ROIs in FDG-PET template space. b Automated ROIs were transferred from $A$ to the original FDG-PET of a 71-year-old, male, AD patient. Note how the ROI's follow the patient rotation and tilting in $\mathbf{b}$ relative to the template image. ROIs: 1 Middle frontal gyrus (MFG), 2 inferior parietal lobule (IP), 3 posterior cingulate cortex (PCC), 4 occipital lobe (OL), 5 prefrontal cortex (PF), 6 superior temporal gyrus (STG), 7 anterior putamen $(\mathrm{APu}), 8$ thalamus $(\mathrm{TH}), 9$ hippocampus (HIP), 10 lateral temporal lobe (LTL), 11 cerebellum. c MNI PET template, singlesubject MNI MRI template and merged template with template ROI
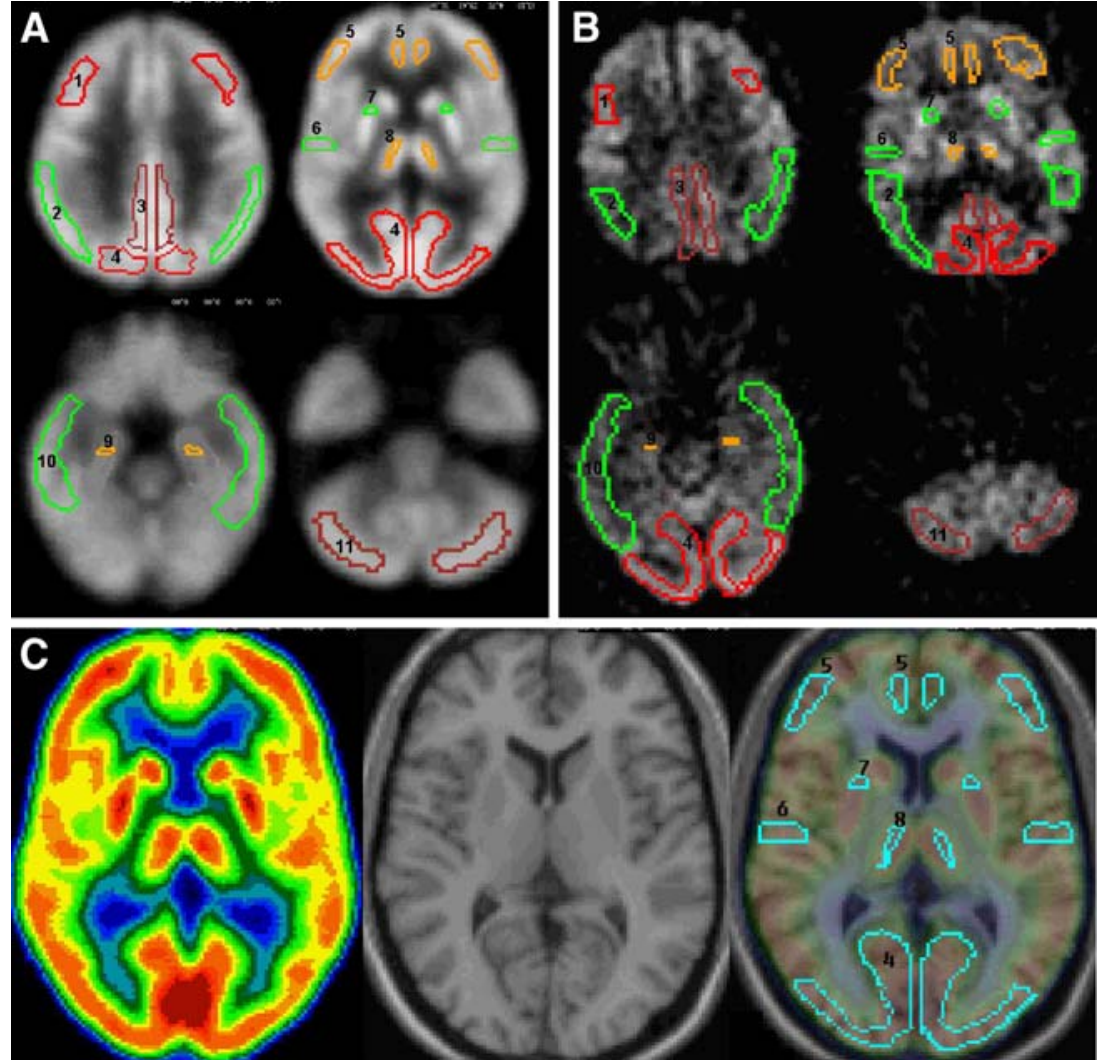

Figure 6

Validating the ROI Template

Methods The anatomical precision and sampling accuracy of the technique was verified on the co-registered MRI and FDG-PET scans of 49 subjects, including 34 NL elderly recruited at NYU and $15 \mathrm{AD}$ patients recruited at th-e University of Turku, Finland. A validation study was also conducted with the PIB-PET scans of the AD patients. Subjects' characteristics are found in Supplemental Table 5. All subjects received an extensive screening and diagnostic battery that consisted of medical, neurological, psychiatric, neuropsychological, and MRI examinations. The NL selected for study had MMSE [11] scores $\geq 28$ and Global Deterioration Scale (GDS) [12] scores of 1 or 2. The AD patients were diagnosed according to the National Institute of Neurological and Communication Disorders and Stroke/ Alzheimer's Disease and Related Disorders Association (NINDS-ADRDA) [13] and DSM-IV criteria [14].

The NYU high-resolution MRI were acquired on a 1.5-T Signa magnet (General Electric, Milwaukee, WI) with a standard whole brain 3D T1-weighted protocol [Radio

Table 5 Subject characteristics of NL and AD in the FDG-PET validation study

\begin{tabular}{|c|c|c|c|c|c|c|}
\hline Subjects & NL & & & $\mathrm{AD}$ & & \\
\hline$N$ & 34 & & & 15 & & \\
\hline Age (years) & 54 (19) $[21-80]$ & & & $72(3)[65-77]$ & & \\
\hline Gender $(\% \mathrm{~F})$ & $69 \%$ & & & $65 \%$ & & \\
\hline MMSE & $29.4(2.6)$ [28-30] & & & $23.6(2.9)$ [17-27] & & \\
\hline $\mathrm{MRglc} \mu \mathrm{mol} / 100 \mathrm{~g} / \mathrm{min}$ & Automated ROIs & Manual ROIs & PVC manual ROIs & Automated ROIs & Manual ROIs & PVC manual ROIs \\
\hline IP & $46.7(7.7)$ & $42.7(6.8)$ & $47.1(7.1)$ & $21.0(4.4)$ & $19.2(4.1)$ & $21.7(4.6)$ \\
\hline MFG & $49.7(9.3)$ & $44.1(7.9)$ & $48.1(8.3)$ & $24.0(5.3)$ & $22.1(4.5)$ & $25.0(5.2)$ \\
\hline PCC & $48.1(8.7)$ & $47.3(8.1)$ & $51.1(8.2)$ & $22.4(4.2)$ & $23.0(5.1)$ & $26.4(5.6)$ \\
\hline STP & $40.7(5.9)$ & $38.4(5.6)$ & $42.1(5.6)$ & $20.4(4.2)$ & $20.1(3.5)$ & $22.7(4.0)$ \\
\hline
\end{tabular}

Values are mean (SD) [range].

$P V C$ Partial volume correction, $I P$ inferior parietal lobe, $M F G$ middle frontal gyrus, $P C C$ posterior cingulate cortex, STG superior temporal gyrus 
Fig. 7 Automatic ROIs shown on a 72-year-old, female, normal control subject. (GDS 2, MMSE 30.) The first row is MRI, and the second row is co-registered FDG-PET. ROIs include MFG, PF, PCC, IP, STG, OL, AP, T, and HIP

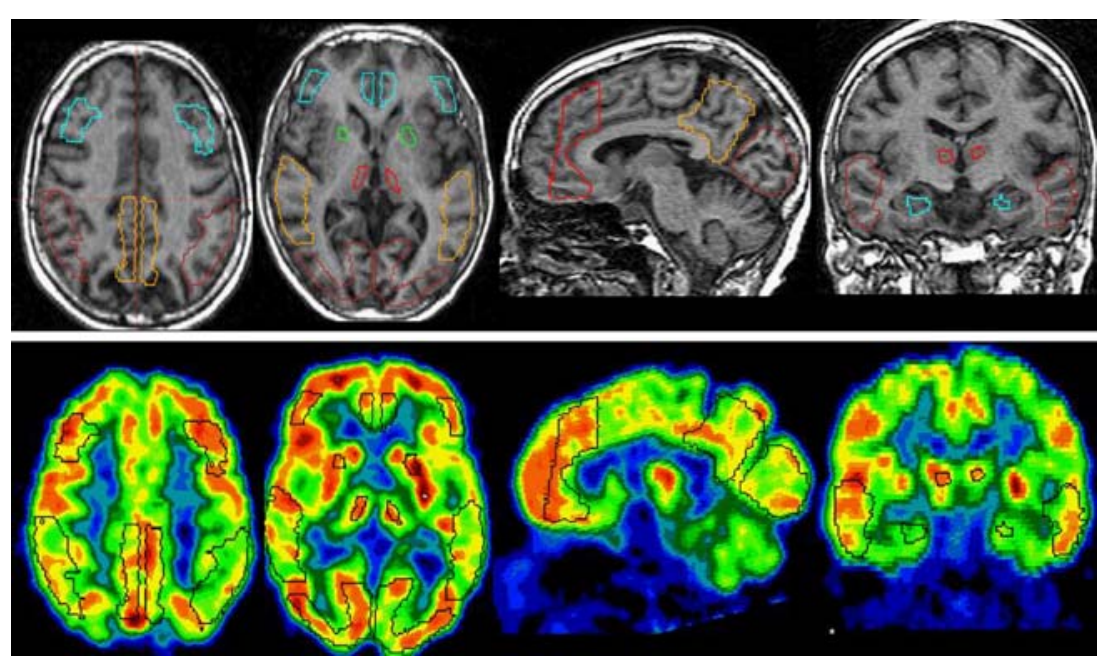

Frequency-Spoiled Gradient Recalled Acquisition in the Steady State (SPGR) pulse sequence, 124 contiguous coronal slices, $1.3 \mathrm{~mm}$ thick, $\mathrm{TR}=35 \mathrm{~ms}, \mathrm{TE}=9 \mathrm{~ms}$, flip angle $60^{\circ}, \mathrm{FOV}=18 \mathrm{~cm}, 1$ excitation, $256 \times 128$ matrix]. Within 3 months of the MRI, an FDG-PET scan was performed at Brookhaven National Laboratories (BNL, Upton, NY, USA) using 2-[18F]fluoro-2-deoxy-D-glucose (FDG) as the tracer on an ECAT EXACT HR+ scanner (Siemens, Knoxville, TN, USA; $3.6 \mathrm{~mm}$ in-plane FWHM, $2.46 \mathrm{~mm}$ slice thickness, $155 \mathrm{~mm}$ axial FOV). Subjects received 3-5 mCi of FDG intravenously, while lying supine in a dimly lit room. PET images were obtained $35 \mathrm{~min}$ after injection and acquired over $20 \mathrm{~min}$. Arterial blood samples were drawn at standard intervals throughout the study, and the absolute CMRglc measures $(\mu \mathrm{mol} / 100 \mathrm{~g} / \mathrm{min})$ were calculated using Sokoloff's model with standard kinetic constants $[15,16]$. Data were reconstructed using filtered back-projection (Fourier rebinning/2D back-projection, Hanning filter with a frequency cutoff of 0.5 cycles/pixel) and corrected for attenuation using $68 \mathrm{Ga} / 68 \mathrm{Ge}$ transmission scans, scatter, and radioactive decay. All the $\mathrm{AD}$ patients $(n=15)$ received FDG and PIB scans at University of Turku, Finland. The scan information can be found in the paper.

Results The anatomical precision and sampling accuracy of the technique was verified on the co-registered MRI and FDG-PET scans from the whole data set of 49 subjects. First, we examined the anatomical fit of the automatic ROIs on MRI by visual inspection of all the scans and all regions. The automated ROIs fit within each anatomic region in all the NL and MCI cases (Fig. 2). For AD, several ROIs were displaced, but none of the ROIs were displaced by more than 4 pixels $(4 \mathrm{~mm})$, which was under the approximate resolution of the PET camera. (i.e., real resolution $>4 \mathrm{~mm}$ FWHM).

\section{Figure 7}

Second, for FDG, we examined the sampling accuracy of the automated ROIs with respect to the gold-standard manual ROI technique with $34 \mathrm{NL}$ and $15 \mathrm{AD}$ subjects. For $\mathrm{PIB}$, the validation was only conducted on $\mathrm{AD}$ subjects. Manual ROIs were drawn on all the MRI of all subjects for four regions (i.e., IP, MFG, STG, and PCC) based on same boundary definition used for the automated ROIs. A twocompartment partial volume (atrophy) correction was applied to the manual ROIs [17]. Regional MRglc ( $\mu \mathrm{mol} /$ $100 \mathrm{~g} / \mathrm{min}$ ) was measured from both the manual and the automated ROIs for all subjects, and hemispheric MRglc means computed. Intra-class correlation coefficients (ICC) and Pearson's correlation coefficients $(r)$ were used to compare the manual to the automated ROI MRglc sampling.

The results for the manual and automated ROI data were highly consistent for all ROIs. There were no significant differences between the Template ROI and manual ROI for either FDG (see Table 1) or PIB (see Table 2). Both before and after atrophy correction for both FDG and PIB, excellent correlations were found between the sampling methods. For the MRglc data, after atrophy correction, the

Table 6 Comparison of manual and automatic template ROIs in the PIB-PET validation study

\begin{tabular}{llll}
\hline $\begin{array}{l}\text { PIB DVR } \\
(\mathrm{n}=15)\end{array}$ & $\begin{array}{l}\text { Automated } \\
\text { ROIs }\end{array}$ & $\begin{array}{l}\text { Manual } \\
\text { ROIs }\end{array}$ & $\begin{array}{l}\text { PVC manual } \\
\text { ROIs }\end{array}$ \\
\hline IP & $1.90(0.52)$ & $1.89(0.46)$ & $2.13(0.52)$ \\
MFG & $2.00(0.52)$ & $1.89(0.45)$ & $2.15(0.50)$ \\
PCC & $2.03(0.56)$ & $2.03(0.62)$ & $2.32(0.68)$ \\
STP & $1.78(0.40)$ & $1.81(0.37)$ & $1.96(0.40)$ \\
\hline
\end{tabular}

Values are mean (SD) [range].

$P V C$ Partial volume correction, $I P$ inferior parietal lobe, $M F G$ middle frontal gyrus, $P C C$ posterior cingulate cortex, $S T G$ superior temporal gyrus 
ICCs ranged from 0.90 superior temporal gyrus (STG) to 0.95 inferior parietal lobule (IP; $p$ 's $<.001)$, with $r$ 's ranging from 0.91 (STG) to 0.98 (IP; $p$ 's $<.001$ ). For PIB data, after atrophy correction, the ICCs ranged between 0.92 (STG) to 0.99 (IP; $p$ 's $<.001$ ), and the $r$ 's ranged between 0.93 (STG) to 0.99 (PCC; $p$ 's $<.001)$.

Conclusions We demonstrate an automated technique that enables one to regionally sample individual brain PET scans with high anatomical precision without requiring an MRI scan for reference or spatial normalization purposes.

\section{References}

1. Selkoe DJ. Alzheimer's disease: genotypes, phenotypes, and treatments. Science 1997;275:630-1.

2. Mirra SS, Heyman A, McKeel D, Sumi SM, Crain BJ, Brownlee LM, et al. The Consortium to Establish a Registry for Alzheimer's Disease (CERAD). Part II. Standardization of the neuropathologic assessment of Alzheimer's disease. Neurology 1991;41:479-86.

3. de Leon MJ, Mosconi L, Blennow K, DeSanti S, Zinkowski R, Mehta PD, et al. Imaging and CSF studies in the preclinical diagnosis of Alzheimer's disease. Ann N Y Acad Sci 2007;1097:114-45.

4. Minoshima S, Giordani B, Berent S, Frey KA, Foster NL, Kuhl DE. Metabolic reduction in the posterior cingulate cortex in very early Alzheimer's disease. Ann Neurol 1997;42:85-94.

5. Nestor PJ, Fryer TD, Smielewski P, Hodges JR. Limbic hypometabolism in Alzheimer's disease and mild cognitive impairment. Ann Neurol 2003;54:343-51.

6. De Santi S, de Leon MJ, Rusinek H, Convit A, Tarshish CY, Roche A, et al. Hippocampal formation glucose metabolism and volume losses in MCI and AD. Neurobiol Aging 2001;22:529-39.

7. Mosconi L, Tsui WH, De Santi S, Li J, Rusinek H, Convit A, et al. Reduced hippocampal metabolism in MCI and AD: automated FDG-PET image analysis. Neurology 2005;64:1860-7.

8. Sokoloff L. Relation between physiological function and energy metabolism in the central nervous system. J Neurochem 1977;29:13-26.

9. Mosconi L. Brain glucose metabolism in the early and specific diagnosis of Alzheimer's disease. FDG-PET studies in MCI and AD. Eur J Nucl Med Mol Imaging 2005;32:486-510.

10. Larrieu S, Letenneur L, Orgogozo JM, et al. Incidence and outcome of mild cognitive impairment in a population-based prospective cohort. Neurology 2002;59:1594-99.

11. Rowe CC, Ng S, Ackermann U, Gong SJ, Pike K, Savage G, et al. Imaging beta-amyloid burden in aging and dementia. Neurology 2007;68:1718-25.

12. Kemppainen NM, Aalto S, Wilson IA, Nagren K, Helin S, Bruck A, et al. PET amyloid ligand 11CPIB uptake is increased in mild cognitive impairment. Neurology 2007;68:1603-06.

13. Verhoeff NP, Wilson AA, Takeshita S, Trop L, Hussey D, Singh $\mathrm{K}$, et al. In-vivo imaging of Alzheimer disease beta-amyloid with 11CSB-13 PET. Am J Geriatr Psychiatry 2004;12:584-95.

14. Klunk WE, Engler H, Nordberg A, Wang Y, Blomqvist G, Holt DP, et al. Imaging brain amyloid in Alzheimer's disease with Pittsburgh Compound-B. Ann Neurol 2004;55:306-19.

15. Delacourte A, David JP, Sergeant N, Buee L, Wattez A, Vermersch $\mathrm{P}$, et al. The biochemical pathway of neurofibrillary degeneration in aging and Alzheimer's disease. Neurology 1999;52:1158-65.

16. Morris JC, Storandt M, McKeel DW Jr, Rubin EH, Price JL, Grant EA, et al. Cerebral amyloid deposition and diffuse plaques in "normal" aging: evidence for presymptomatic and very mild Alzheimer's disease. Neurology 1996;46:707-19.

17. Thal DR, Rub U, Orantes M, Braak H. Phases of A betadeposition in the human brain and its relevance for the development of AD. Neurology 2002;58:1791-800.

18. Braak H, Braak E. Neuropathological stageing of Alzheimerrelated changes. Acta Neuropathol (Berl) 1991;82:239-59.

19. Bennett DA, Schneider JA, Tang Y, Arnold SE, Wilson RS. The effect of social networks on the relation between Alzheimer's disease pathology and level of cognitive function in old people: a longitudinal cohort study. Lancet Neurol 2006;5:406-12.

20. McKhann G, Drachman D, Folstein M, Katzman R, Price D, Stadlan EM. Clinical diagnosis of Alzheimer's disease: report of the NINCDS-ADRDA Work Group under the auspices of Department of Health and Human Services Task Force on Alzheimer's Disease. Neurology 1984;34:939-44.

21. American Psychiatric Association. Diagnostic and Statistical Manual of Mental Disorders, Fourth Edition (DSM-IV). Washington, DC: American Psychiatric Association; 1994. p. 143-7.

22. Cockrell JR, Folstein MF. Mini-Mental State Examination (MMSE). Psychopharmacol Bull 1988;24:689-92.

23. Petersen RC, Doody R, Kurz A, Mohs RC, Morris JC, Rabins PV, et al. Current concepts in mild cognitive impairment. Arch Neurol 2001;58:1985-92.

24. Logan J, Fowler JS, Volkow ND, Wolf AP, Dewey SL, Schlyer DJ, et al. Graphical analysis of reversible radioligand binding from time-activity measurements applied to N-11C-methyl-(-)-cocaine PET studies in human subjects. J Cereb Blood Flow Metab 1990;10:740-7.

25. Lopresti BJ, Klunk WE, Mathis CA, Hoge JA, Ziolko SK, Lu X, et al. Simplified quantification of Pittsburgh Compound B amyloid imaging PET studies: a comparative analysis. J Nucl Med 2005;46:1959-72.

26. Patlak CS, Blasberg RG, Fenstermacher JD. Graphical evaluation of blood-to-brain transfer constants from multiple-time uptake data. J Cereb Blood Flow Metab 1983;3:1-7.

27. Woods RP, Mazziotta JC, Cherry SR. MRI-PET registration with automated algorithm. J Comput Assist Tomogr 1993;17:536-46.

28. Tsui WH, Rusinek H, Van Gelder P, Lebedev S. Analyzing multimodality tomographic images and associated regions of interest with MIDAS. Proc SPIE Med Imaging: Image Processing 2001;4322:1725-34.

29. Woods RP, Grafton ST, Watson JD, Sicotte NL, Mazziotta JC. Automated image registration: II. Intersubject validation of linear and nonlinear models. J Comput Assist Tomogr 1998;22:153-65.

30. Good CD, Johnsrude IS, Ashburner J, Henson RN, Friston KJ, Frackowiak RS. A voxel-based morphometric study of ageing in 465 normal adult human brains. Neuroimage 2001;14:21-36.

31. Benson DF, Kuhl DE, Hawkins RA, Phelps ME, Cummings JL, Tsai SY. The fluorodeoxyglucose $18 \mathrm{~F}$ scan in Alzheimer's disease and multi-infarct dementia. Arch Neurol 1983;40:711-4.

32. Joachim CL, Morris JH, Selkoe DJ. Diffuse senile plaques occur commonly in the cerebellum in Alzheimer's disease. Am J Pathol 1989;135:309-19.

33. Klunk WE, Wang Y, Huang GF, Debnath ML, Holt DP, Shao L, et al. The binding of 2-(4'-methylaminophenyl)benzothiazole to postmortem brain homogenates is dominated by the amyloid component. J Neurosci 2003;23:2086-92.

34. Ziolko SK, Weissfeld LA, Klunk WE, Mathis CA, Hoge JA, Lopresti BJ, et al. Evaluation of voxel-based methods for the statistical analysis of PIB PET amyloid imaging studies in Alzheimer's disease. Neuroimage 2006;33:94-102. 
35. Edison P, Archer HA, Hinz R, et al. Amyloid, hypometabolism, and cognition in Alzheimer's disease: An [11C]PIB and [18F] FDG PET study. Neurology. 2007;68:501-8.

36. Ng S, Villemagne VL, Berlangieri S, et al. Visual assessment versus quantitative assessment of 11C-PIB PET and 18F-FDG PET for detection of Alzheimer's disease. J Nucl Med. 2007;48:547-52.

37. de Leon MJ, Convit A, Wolf OT, Tarshish CY, DeSanti S, Rusinek $\mathrm{H}$, et al. Prediction of cognitive decline in normal elderly subjects with 2-(18)Ffluoro-2-deoxy-D-glucose/poitron-emission tomography (FDG/PET). Proc Natl Acad Sci U S A 2001;98: 10966-71.

38. Nestor PJ, Fryer TD, Ikeda M, Hodges JR. Retrosplenial cortex (BA 29/30) hypometabolism in mild cognitive impairment (prodromal Alzheimer's disease). Eur J Neurosci 2003;18: 2663-7.

39. Drzezga A, Lautenschlager N, Siebner H, Riemenschneider M, Willoch F, Minoshima S, et al. Cerebral metabolic changes accompanying conversion of mild cognitive impairment into Alzheimer's disease: a PET follow-up study. Eur J Nucl Med Mol Imaging 2003;30:1104-13.

40. Minoshima S, Foster NL, Kuhl DE. Posterior cingulate cortex in Alzheimer's disease. Lancet 1994;344:895.

41. Chen PY, Popovich PM. Correlation : parametric and nonparametric measures. Sage Pubns; 2002 Jan.

42. Friston KJ, Frith CD, Liddle PF, Frackowiak RS. Comparing functional (PET) images: the assessment of significant change. J Cereb Blood Flow Metab 1991;11:690-9. 